\section{Protonenaffinität und Basizität von Alkylbenzolen}

\section{H.-D. Klotz, H. Drost und W. Schulz}

Deutsche Akademie der Wissenschaften zu Berlin, Forschungsgemeinschaft, Institut für Biophysik, Berlin-Buch, DDR

(Z. Naturforsch. 23 a, 1690-1691 [1968]; eingeg. am 10. November 1967)

Nach der Theorie von BRönsted ${ }^{1}$ wird die SäureBasen-Wechselwirkung in Lösung als eine protolytische Reaktion der Art

$$
\text { Säure } \rightleftarrows \text { Base }+ \text { Proton }
$$

angesehen. Die Säure wirkt dabei als Protonendonator, die korrespondierende Base als Protonenakzeptor. Säure und Base bilden zusammen ein protolytisches Reaktionssystem, dessen Gleichgewichtskonstanten $K_{\mathrm{A}}$ bzw. $K_{\mathrm{B}}$ - die sogen. Aziditäts- bzw. Basizitätskonstanten durch die Beziehung

$$
K_{\mathrm{A}}=a_{\mathrm{H}^{+}} \frac{a_{\text {Base }}}{a_{\text {Säure }}} \quad \text { bzw. } \quad K_{\mathrm{B}}=\frac{1}{K_{\mathrm{A}}}=\frac{1}{a_{\mathrm{H}^{+}}} \cdot \frac{a_{\text {Säure }}}{a_{\text {Base }}}
$$

charakterisiert werden können. $K_{\mathrm{A}}$ bzw. $K_{\mathrm{B}}$ sind im allgemeinen der Messung nicht direkt zugänglich, da das Lösungsmittel meist selbst ein Säure-Basen-Paar darstellt, das sich dem ursprünglichen Reaktionssystem überlagert. Ein besonders großer Einfluß des Lösungsmittels auf das Säure-Basen-Gleichgewicht ergibt sich für den Fall organischer Substanzen. Die Einbeziehung organischer Substanzen in die Brönstedsche Theorie, d. h. ihre Definition als Säuren oder Basen, erweist sich als sinnvoll, da viele von ihnen, vor allem die ungesättigten Verbindungen, in verschiedenen Lösungsmitteln stabile Protonen-Additions- oder $\pi$-Komplexe, entsprechend der Reaktion $\mathrm{HX}+\mathrm{A} \rightleftharpoons \mathrm{AH}^{+}+\mathrm{X}^{-}$bilden. Damit wäre ihnen nach BRöNsted als Protonenakzeptoren ein basischer Charakter zuzuordnen; gleichzeitig käme dem $\pi$-Komplex die Bedeutung einer zur ungesättigten organischen Verbindung konjugierten kationischen Säure zu. Die Untersuchung der Bildung von ProtonAdditionskomplexen $\mathrm{AH}^{+}$organischer Verbindungen erfolgt meist unter speziellen Bedingungen, z. B. in sogen. ternären Systemen, bestehend aus der aromatischen Substanz A, einer Lewis-Säure $\mathbf{M X}_{\mathbf{3}}$ und einer Wasserstoff-Halogen-Verbindung HX, wodurch zwangsläufig die Gleichgewichtskonstante $K_{\mathrm{B}}$ vorwiegend das gesamte System charakterisiert. Hinzu kommt, daß auch der Komplex $\mathrm{AH}^{+}$selbst durch das Lösungsmittel beeinflußt wird. Während es z. B. in starken Säuren infolge der starken Wechselwirkung zwischen den Reaktionspartnern zur Bildung von echten, durch eine kovalente Bindung ausgezeichneten Komplexen kommt, entstehen in schwachen Lösungsmitteln Chargetransfer-

1 I. N. Brönsted, Rec. Trav. Chim. 42, 718 [1932].

2 H. H. Perkampus, Adv. Phys. Org. Chem. 4, 195 [1966].

3 V. L. Talrose u. E. L. Frankevich, Dokl. Akad. Nauk USSR 111, 376 [1956].

${ }_{4} D$ bedeutet Dissoziationsenergie und $I_{P}$ Ionisationspotential.
Komplexe, die durch keine am Molekül lokalisierbare Bindung zusammengehalten werden ${ }^{2}$. Damit erfährt die Basizitätskonstante eine weitere Relativierung, wodurch sie nur in geringem Maße zur Charakterisierung der Basizität einer organischen Verbindung bzw. zur Verifizierung einer Säure-Base-Theorie geignet sein dürfte. Schwierigkeiten dieser Art können jedoch vermieden werden, falls es gelingt, die Basizität als molekülspezifische Größe zu definieren, d.h. die Basizitätskonstante $K_{\mathrm{B}}$ auf die Molekülparameter der in Frage stehenden organischen Verbindung zurückzuführen.

Als wesentliche Voraussetzung für die Realisierung dieses Vorhabens erschien uns die Reduzierung des komplizierten protolytischen Reaktionssystems (1) auf den Elementarprozeß der Protonenübertragung

$$
\mathrm{A}+\mathrm{H}^{+} \rightarrow \mathrm{AH}^{+}+\Delta E \text {. }
$$

Dabei bedeuten A die Brönsted-Base und $\Delta E$ ihre Protonenaffinität, $d . h$. die bei der Protonenanlagerung an A freiwerdende Energie. Da die Protonenaffinität $\Delta E$ der Bindungsfestigkeit des Protons im Komplex $\mathrm{AH}^{+}$ entspricht und damit lediglich von der Struktur des Moleküls A bestimmt ist, erschien es sinnvoll, sie auch als quantitatives $\mathrm{Ma}$ f für die Basizität des Moleküls A anzusehen. Dadurch ergäbe sich die Möglichkeit, das protolytische Reaktionsverhalten ungesättigter organischer Verbindungen mit einer aus ihrer Molekülstruktur folgenden Größe zu verknüpfen. Der Anspruch derartiger Vorstellung auf Gültigkeit hängt wesentlich von der Kenntnis der einzelnen $\Delta E$-Werte und ihrer Beziehung zu den bisher ermittelten $K_{\mathrm{B}}$-Konstanten ab. Praktisch läuft dieses Vorhaben auf die Bestimmung der meist unbekannten $\Delta E$-Werte organischer Substanzen hinaus.

Bei den folgenden Untersuchungen wurde die Protonenaffinität mit Hilfe von Protonentransfer-Reaktionen des Typs

$$
\mathrm{RH}^{+}+\mathrm{A} \rightarrow \mathrm{AH}^{+}+\mathrm{R}
$$

bestimmt. Wenn $R$ eine schwache BröNSTED-Base ist, so ist $\mathrm{RH}^{+}$eine starke BRönsted-Säure, und ein Protonentransfer von $\mathrm{RH}^{+}$zu A findet statt, falls A eine stärkere Base als $R$ ist. Nach Talrose und Frankevich ${ }^{3}$ läßt sich aus (3) die Protonenaffinität von A entsprechend ${ }^{4}$

$$
\begin{array}{r}
D\left(\mathrm{R}_{1}-\mathrm{H}\right)+I_{\mathrm{P}}(\mathrm{H})-I_{\mathrm{P}}\left(\mathrm{R}_{1} \mathrm{H}\right)<\Delta E(\mathrm{~A}) \\
<D\left(\mathrm{R}_{\mathbf{2}}-\mathrm{H}\right)+I_{\mathrm{P}}(\mathrm{H})-I_{\mathrm{P}}\left(\mathrm{R}_{\mathbf{2}} \mathrm{H}\right)
\end{array}
$$

einschachteln, je nachdem, ob Reaktion (3) mit dem Partner $\mathrm{R}_{1} \mathrm{H}^{+}$abläuft oder mit $\mathrm{R}_{2} \mathrm{H}^{+}$nicht nachweisbar ist.

Die Untersuchungen erfolgten an einer Reihe von Methylbenzolen, deren Basizitätskonstanten $K_{\mathrm{B}}{ }^{5}$, sowie die Werte für die relative Basizität ${ }^{6}$ gut bekannt

5 M. Kilpatrick u. F. E. Lubovsky, J. Amer. Chem. Soc. 75, 577 [1953].

6 H. C. Brown u. J. P. Brady, J. Amer. Chem. Soc. 74, 3570 [1952]. 


\begin{tabular}{|c|c|c|c|c|c|c|}
\hline \multirow[b]{2}{*}{ Alkylbenzol } & \multicolumn{2}{|c|}{$\Delta E[\mathrm{eV}]$} & \multirow{2}{*}{$\begin{array}{c}\text { Reaktionen, mit denen die untere bzw. } \\
\text { die obere Grenze von } \Delta E \text { bestimmt } \\
\text { wurde }\end{array}$} & \multirow{2}{*}{$\begin{array}{c}K_{\mathrm{B}} \\
\left(\text { s. Anm. }{ }^{4}\right)\end{array}$} & \multirow{2}{*}{$\begin{array}{l}\text { relative } \\
\text { Basizität * } \\
\left(\mathrm{s} . \text { Anm. }{ }^{5}\right)\end{array}$} & \multirow{2}{*}{$\begin{array}{l}\text { relative } \\
\text { Reaktivität * } \\
\text { (s. Anm. }{ }^{5} \text { ) }\end{array}$} \\
\hline & $\begin{array}{l}\text { untere } \\
\text { Grenze }\end{array}$ & $\begin{array}{c}\text { obere } \\
\text { Grenze }\end{array}$ & & & & \\
\hline $\mathrm{C}_{6} \mathrm{H}_{6}$ & 6,48 & 7,07 & 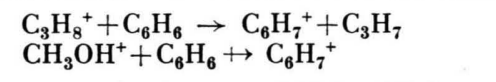 & $1,05 \cdot 10^{-8}$ & 0,61 & $5,0 \cdot 10^{-4}$ \\
\hline $\mathrm{C}_{6} \mathrm{H}_{5} \mathrm{CH}_{3}$ & 7,07 & 7,6 & 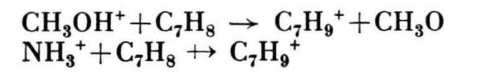 & $2,0 \cdot 10^{-7}$ & 0,92 & $1,6 \cdot 10^{-1}$ \\
\hline $1,2\left(\mathrm{CH}_{3}\right)_{2} \mathrm{C}_{6} \mathrm{H}_{4}$ & 7,28 & 7,9 & $\begin{array}{l}\mathrm{H}_{2} \mathrm{~S}^{+}+\mathrm{C}_{8} \mathrm{H}_{10} \rightarrow \mathrm{C}_{8} \mathrm{H}_{11}^{+}+\mathrm{HS} \\
\mathrm{C}_{8} \mathrm{H}_{10}^{+}+\mathrm{C}_{8} \mathrm{H}_{10} \rightarrow \mathrm{C}_{8} \mathrm{H}_{11}^{+}\end{array}$ & $5,8 \cdot 10^{-7}$ & 1,13 & $2 \cdot 10$ \\
\hline $1,3,5\left(\mathrm{CH}_{3}\right)_{3} \mathrm{C}_{6} \mathrm{H}_{3}$ & 8,14 & 8,84 & $\begin{array}{l}\mathrm{C}_{7} \mathrm{H}_{8}^{+}+\mathrm{C}_{9} \mathrm{H}_{12} \rightarrow \mathrm{C}_{9} \mathrm{H}_{13}^{+}+\mathrm{C}_{7} \mathrm{H}_{7} \\
\mathrm{C}_{6} \mathrm{H}_{6}^{+}+\mathrm{C}_{9} \mathrm{H}_{12} \rightarrow \mathrm{C}_{9} \mathrm{H}_{13}^{+}\end{array}$ & $3,2 \cdot 10^{-3}$ & 1,59 & $8,0 \cdot 10^{4}$ \\
\hline $1,2,4,5\left(\mathrm{CH}_{3}\right)_{4} \mathrm{C}_{6} \mathrm{H}_{2}$ & 8,84 & 10,7 & $\begin{array}{l}\mathrm{C}_{6} \mathrm{H}_{6}^{+}+\mathrm{C}_{10} \mathrm{H}_{14} \rightarrow \mathrm{C}_{10} \mathrm{H}_{15}^{+}+\mathrm{C}_{6} \mathrm{H}_{5} \\
\mathrm{C}_{10} \mathrm{H}_{14}^{+}+\mathrm{H}_{2} \mathrm{O} \rightarrow \mathrm{C}_{10} \mathrm{H}_{15}^{+}\end{array}$ & $3,8 \cdot 10^{-3}$ & 1,67 & $2,4 \cdot 10^{5}$ \\
\hline
\end{tabular}

* p-Xylol $=1$.

Tab. 1 .

sind. Das Ergebnis der Untersuchungen ist in Tab. 1 bzw. Abb. 1 zusammengestellt. Tab. 1 enthält die auf Grund der angeführten Reaktionsgleichung (3) ermittelten Protonenaffinitäten $\Delta E$ (jeweils deren obere bzw. untere Grenze) zusammen mit den aus der Literatur entnommenen $K_{\mathrm{B}}$-Konstanten, den relativen Basizitätswerten, sowie den Werten für die relative Reaktivität der einzelnen Methylbenzole. Der erwartete qualitative Zusammenhang zwischen allen diesen unter den Bedingungen eines protolytischen Systems, d. h. in Lösung bestimmten Konstanten und der das einzelne Molekül charakterisierenden Protonenaffinität $\Delta E$ ist deutlich zu erkennen. Mit zunehmender Methylierung steigen sowohl die Protonenaffinität als auch die verschiedenen Konstanten an. Der lineare Kurvenverlauf in Abb. 1 unterstreicht diesen Zusammenhang für den Fall der Beziehung Protonenaffinität $\Delta E$-relative Basizität zusätzlich. Wir sehen in den Ergebnissen unserer Untersuchungen eine erste Bestätigung für die prinzipielle Möglichkeit, das Basizitätsverhalten vor organischen Verbindungen durch molekulare Parameter, in diesem Fall durch die Protonenaffinität, zu charakterisieren.

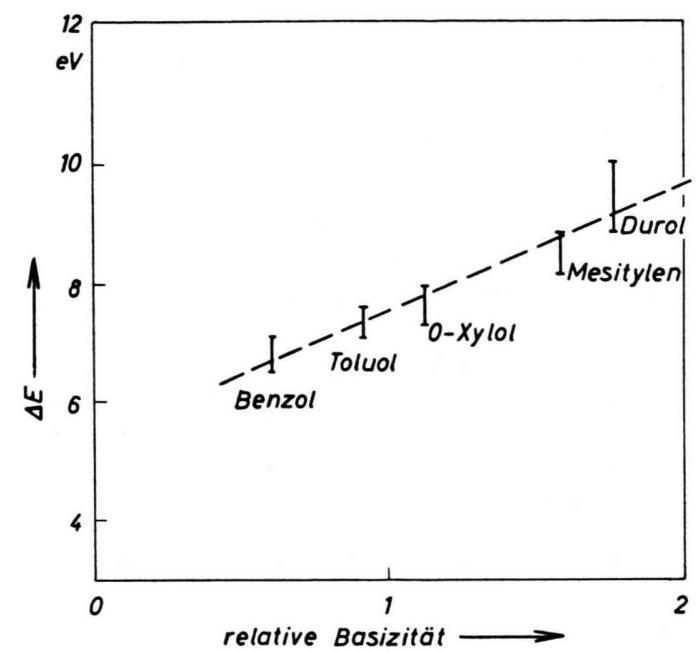

Abb. 1. Zusammenhang von Protonenaffinität $\Delta E$ und relativer Basizität (vgl. Tab. 1) in Abhängigkeit von der Anzahl der Methylgruppen am Benzol. 